\title{
Prostaglandin $\mathbf{E}_{\mathbf{2}}$ and Polyenylphosphatidylcholine: Stiff Competition for the Fibrotic Complications of Inflammatory Bowel Disease?
}

\author{
Steven K. Huang ${ }^{1}$ Marc Peters-Golden ${ }^{1}$
}

Received: 2 April 2015/Accepted: 10 April 2015/Published online: 24 April 2015

(C) Springer Science+Business Media New York 2015

\begin{abstract}
Although considerable progress has been made in the treatment of inflammatory bowel disease (IBD), more than $75 \%$ of patients with Crohn's disease still require surgery at least once in their lifetime, usually for strictures and bowel obstruction. These often reflect intestinal fibrosis, a common histopathologic feature of IBD [1]. Although intestinal fibrosis is traditionally considered a consequence of excessive chronic inflammation, treatment with anti-tumor necrosis factor- $\alpha$ and other immunomodulatory drugs, which effectively ameliorate bowel inflammation, has unfortunately done little to curb the incidence of fibrotic complications. This observation has motivated investigators to reconsider the mechanisms that lead to intestinal fibrosis in an effort to identify alternative therapeutic approaches [2]. In this issue of Digestive Diseases and Sciences, Baird et al. [3] report on the anti-fibrotic potential of prostaglandin $\mathrm{E}_{2}\left(\mathrm{PGE}_{2}\right)$ and polyenylphosphatidylcholine (PPC).
\end{abstract}

The initial glimmer of our current recognition that $\mathrm{PGE}_{2}$ is critical to the homeostasis of the gastrointestinal (GI) tract dates to 1938, when acetylsalicylic acid, or aspirin, was first reported to cause gastric hemorrhage [4], which in 1955 was attributed to its potential to promote erosive gastritis [5]. The roots of our mechanistic understanding for these observations derive from two Nobel Prize-winning discoveries, namely the purification and structural characterization of prostaglandins by Sune Bergström and Bengt

Marc Peters-Golden

petersm@umich.edu

1 Division of Pulmonary and Critical Care Medicine, University of Michigan Hospital and Health Systems, 6301 Medical Sciences Research Building III, 1150 W. Medical Center Drive, Ann Arbor, MI 48109-5642, USA
Samuelsson, and the subsequent discovery by John Vane that aspirin inhibited the enzymatic production of prostaglandins. Today, it is recognized that abundant production of $\mathrm{PGE}_{2}$ by the constitutively active cyclooxygenase- 1 in gastric epithelial cells is critical to their protection from a harsh acidic environment. It is now appreciated that $\mathrm{PGE}_{2}$ promotes epithelial integrity in other parts of the GI tract and indeed in other organs. That $\mathrm{PGE}_{2}$ protects against epithelial injury is evident from its anti-apoptotic effects in a mouse model of radiation colitis [6]. Although $\mathrm{PGE}_{2}$ is classically thought of as a pro-inflammatory molecule, this reputation largely reflects its actions on the microvasculature, but-interestingly-its effects on leukocytes are predominantly suppressive, as exemplified by its contribution to immune tolerance in the gut [7]. The increased risk of Crohn's disease associated with the use of aspirin and other NSAIDs [8] may therefore be explained by the loss of both the anti-inflammatory and epithelial-protective actions of $\mathrm{PGE}_{2}$.

Returning to the challenge of curbing fibrotic responses, significant data-mostly from studies of the lung, liver, kidney, and skin - support the hypothesis that $\mathrm{PGE}_{2}$ exerts anti-fibrotic effects independently of its anti-inflammatory and epithelial-protective actions. This reflects that $\mathrm{PGE}_{2}$ can also inhibit nearly all aspects of fibroblast activation via its ability to increase intracellular cyclic AMP [9]; in vivo administration of $\mathrm{PGE}_{2}$ can prevent lung fibrosis in mouse models [10]. The paper by Baird and colleagues reports for the first time that exogenous administration of $\mathrm{PGE}_{2}$ ameliorated intestinal fibrosis in the commonly employed 2,4,6-trinitrobenzene sulfonic acid (TNBS) murine model. The authors also examined the effects of $\mathrm{PGE}_{2}$ on intestinal fibroblasts in vitro, and like fibroblasts from other organs, $\mathrm{PGE}_{2}$ directly inhibited fibroblast proliferation and collagen production. Since in this in vivo study $\mathrm{PGE}_{2}$ was 
co-administered with TNBS, it inhibited intestinal inflammation as well. This experimental design, therefore, fails to distinguish whether $\mathrm{PGE}_{2}$ is capable of actually reversing preexisting intestinal fibrosis or whether it merely limits the inflammatory damage that culminates in fibrosis. As noted earlier, an independent anti-fibrotic effect is essential if we are to argue that $\mathrm{PGE}_{2}$ is superior to existing immunomodulatory drugs used to treat IBD. Although its recognized direct inhibitory effects on fibroblast functions would predict that this would be the case, a proof-ofprinciple experiment would require its administration later in the disease model when intestinal fibrosis is already established.

What about PPC? PPC is a mixture of polyunsaturated phosphatidylcholine (PC) molecules derived from plantbased extracts that has primarily been used for the treatment of liver disease [11]. PC, an essential component of the lipid membrane bilayers of all cells, contributes to the integrity of the mucosal barrier of epithelial cells, including those lining the GI tract. The observation that mucosal PC content is diminished in patients with IBD [12] prompted early-stage clinical trials that suggest that exogenous PPC is potentially beneficial for IBD patients [13]. Baird and colleagues reported that PPC inhibited intestinal inflammation and fibrosis elicited by TNBS to the same degree as did $\mathrm{PGE}_{2}$, consistent with prior reports of the inhibitory effects of PPC on alcohol-induced cirrhosis in vivo [11] and on collagen synthesis by hepatic stellate fibroblast-like cells in vitro [14]. The parallel actions of PPC and $\mathrm{PGE}_{2}$ led to the hypothesis that the actions of PPC may be mediated by $\mathrm{PGE}_{2}$, which was not supported by the observation that colonic tissue concentrations of $\mathrm{PGE}_{2}$ did not increase with systemic administration of PPC. A more rigorous approach to this question might be to test whether the protective actions of PPC are abolished either by pharmacologic or genetic inhibition of $\mathrm{PGE}_{2}$ biosynthesis or by antagonism or deletion of its receptors. It is alternatively possible that PPC could initiate the production of other anti-inflammatory, anti-fibrotic eicosanoids that increase intracellular cyclic AMP (e.g., prostaglandin $\mathrm{D}_{2}$ or $\mathrm{I}_{2}$ ) that the authors did not measure. As was noted above for $\mathrm{PGE}_{2}$, addressing the question of whether PPC can attenuate intestinal fibrosis in this model independent of its effects on inflammation is also worthy of future study. Certainly, this work provides justification for additional exploration of PPC as potential therapy in IBD.

While acknowledging the promise of $\mathrm{PGE}_{2}$ and $\mathrm{PPC}$ in treating intestinal fibrosis, questions and challenges still remain. In the study by Baird and colleagues, $\mathrm{PGE}_{2}$ was administered orally, which brings to mind an oral analog of $\mathrm{PGE}_{2}$, misoprostol $\left(\right.$ Cytotec $\left.^{\circledR}\right)$, which has long been available to protect the stomach from NSAID-induced injury but whose use is notably limited by accompanying diarrhea. A potential strategy to circumvent this limitation would be to take advantage of the functional specificity mediated by individual receptors for $\mathrm{PGE}_{2}$. There are four such E prostanoid receptors, termed EP1-EP4, with differing cellular distribution and signal transduction mechanisms; only EP2 and EP4 signal via increased cyclic AMP. Increases in intestinal motility are mediated by EP1 and EP3 [15], whereas a selective EP4 agonist protects against colitis manifestations in a mouse model while enhancing epithelial survival and regeneration [16]. Selective agonists of EP2 and/or EP4 may thus offer the promise of anti-inflammatory, epithelial-protective, and anti-fibrotic actions - ideal for IBD — without the unwanted side effects inherent to $\mathrm{PGE}_{2}$ itself or its receptor-nonselective analogs like misoprostol. One further possible challenge that might be anticipated is resistance to the beneficial effects of $\mathrm{PGE}_{2}$, as has been identified in other fibrotic disorders [17]. Finally, we would be remiss if we failed to acknowledge the well-recognized ability of $\mathrm{PGE}_{2}$ to promote tumorigenesis in the colon [18] and elsewhere-an important issue for IBD patients. It appears that several EP receptors may contribute to tumorigenesis at different stages of the disease. Therefore, a better understanding of whether and how the potential benefits of selective EP agonists can be harnessed without promoting tumor formation would be essential going forward. The question of whether PPC promotes tumorigenesis in this setting would also be important to answer. The promising results that Baird and colleagues present in this issue suggest that the answers to these questions are worth pursuing.

\section{References}

1. Pittet V, Rogler G, Michetti P, et al. Penetrating or stricturing diseases are the major determinants of time to first and repeat resection surgery in Crohn's disease. Digestion. 2013;87:212-221.

2. Rogler G. New therapeutic avenues for treatment of fibrosis: can we learn from other diseases? Dig Dis. 2014;32(Suppl 1):39-49.

3. Baird AC, Lloyd F, Lawrance IC. Prostaglandin $\mathrm{E}_{2}$ and polyenylphosphatidylcholine protect against intestinal fibrosis and regulate myofibroblast function. Dig Dis Sci. (Epub ahead of print). doi: 10.1007/s10620-015-3552-9.

4. Douthwaite AH. Recent advances in medical diagnosis and treatment. Br Med J. 1938;1:1143-1146.

5. Muir A, Cossar IA. Aspirin and ulcer. Br Med J. 1955;2:7-12.

6. Tessner TG, Muhale F, Riehl TE, Anant S, Stenson WF. Prostaglandin $E_{2}$ reduces radiation-induced epithelial apoptosis through a mechanism involving AKT activation and bax translocation. J Clin Investig. 2004;114:1676-1685.

7. Chinen T, Komai K, Muto G, et al. Prostaglandin $\mathrm{E}_{2}$ and SOCS1 have a role in intestinal immune tolerance. Nat Commun. 2011;2:190.

8. Felder JB, Korelitz BI, Rajapakse R, Schwarz S, Horatagis AP, Gleim G. Effects of nonsteroidal antiinflammatory drugs on inflammatory bowel disease: a case-control study. Am J Gastroenterol. 2000;95:1949-1954. 
9. Huang S, Wettlaufer SH, Hogaboam C, Aronoff DM, PetersGolden M. Prostaglandin $\mathrm{E}_{2}$ inhibits collagen expression and proliferation in patient-derived normal lung fibroblasts via $\mathrm{E}$ prostanoid 2 receptor and cAMP signaling. Am J Physiol Lung Cell Mol Physiol. 2007;292:L405-L413.

10. Dackor RT, Cheng J, Voltz JW, et al. Prostaglandin $E_{2}$ protects murine lungs from bleomycin-induced pulmonary fibrosis and lung dysfunction. Am J Physiol Lung Cell Mol Physiol. 2011; 301:L645-L655.

11. Lieber CS. Prevention and treatment of liver fibrosis based on pathogenesis. Alcohol Clin Exp Res. 1999;23:944-949.

12. Ehehalt R, Wagenblast J, Erben G, et al. Phosphatidylcholine and lysophosphatidylcholine in intestinal mucus of ulcerative colitis patients. A quantitative approach by nanoelectrospray-tandem mass spectrometry. Scand J Gastroenterol. 2004;39:737-742.

13. Stremmel W, Braun A, Hanemann A, Ehehalt R, Autschbach F, Karner M. Delayed release phosphatidylcholine in chronic-active ulcerative colitis: a randomized, double-blinded, dose finding study. J Clin Gastroenterol. 2010;44:e101-e107.
14. Cao Q, Mak KM, Lieber CS. Dilinoleoylphosphatidylcholine prevents transforming growth factor-beta1-mediated collagen accumulation in cultured rat hepatic stellate cells. J Lab Clin Med. 2002;139:202-210.

15. Dey I, Lejeune M, Chadee K. Prostaglandin $E_{2}$ receptor distribution and function in the gastrointestinal tract. Br J Pharmacol. 2006;149:611-623.

16. Jiang GL, Nieves A, Im WB, Old DW, Dinh DT, Wheeler L. The prevention of colitis by E Prostanoid receptor 4 agonist through enhancement of epithelium survival and regeneration. $J$ Pharmacol Exp Ther. 2007;320:22-28.

17. Huang SK, Wettlaufer SH, Hogaboam CM, et al. Variable prostaglandin $\mathrm{E}_{2}$ resistance in fibroblasts from patients with usual interstitial pneumonia. Am J Respir Crit Care Med. 2008;177: 66-74.

18. Wang D, Dubois RN. Prostaglandins and cancer. Gut. 2006;55: $115-122$. 